\title{
Sensitivity to Error During Visuomotor Adaptation is Similarly Modulated by Abrupt, Gradual and Random Perturbation Schedules
}

${ }^{1}$ Graduate Program in Neuroscience, Western University, London, ON, Canada

$7 \quad 2$ Brain and Mind Institute, Western University, London, ON, Canada

$8 \quad{ }^{3}$ Department of Psychology, Western University, London, ON, Canada

$9{ }^{4}$ Donders Institute for Brain, Cognition and Behaviour, Radboud University, Nijmegen, The 10 Netherlands

$11{ }^{5}$ Dept. of Human Movement Sciences, Vrije Universiteit Amsterdam, Amsterdam, The

12 Netherlands

$13{ }^{6}$ Department of Physiology and Pharmacology, Schulich School of Medicine \& Dentistry,

14 Western University, London, ON, Canada

$15 \quad{ }^{7}$ Haskins Laboratories, New Haven CT, USA

Acknowledgements: Canadian Institutes of Health Research (CIHR) and the Natural Sciences

The authors declare no competing financial interests.

Please Address Correspondence to:

Paul L. Gribble

30 Brain and Mind Institute, Dept. of Psychology

31 Western University

321151 Richmond St.,

33 London, ON, Canada, N6A 3K7

34 Email: pgribble@uwo.ca 


\section{Abstract}

39 It has been suggested that sensorimotor adaptation involves at least two processes (i.e., fast and

40 slow) that differ in retention and error sensitivity. Previous work has shown that repeated

41 exposure to an abrupt force field perturbation results in greater error sensitivity for both the fast

42 and slow processes. While this implies that the faster relearning is associated with increased

43 error sensitivity, it remains unclear what aspects of prior experience modulate error sensitivity.

44 In the present study, we manipulated the initial training using different perturbation schedules,

45 thought to differentially affect fast and slow learning processes based on error magnitude, and

46 then observed what effect prior learning had on subsequent adaptation. During initial training of

47 a visuomotor rotation task, we exposed three groups of participants to either an abrupt, a gradual,

48 or a random perturbation schedule. During a testing session, all three groups were subsequently

49 exposed to an abrupt perturbation schedule. Comparing the two sessions of the control group

50 who experienced repetition of the same perturbation, we found an increased error sensitivity for

51 both processes. We found that the error sensitivity was increased for both the fast and slow

52 processes, with no reliable changes in the retention, for both the gradual and structural learning

53 groups when compared to the first session of the control group. We discuss the findings in the

54 context of how fast and slow learning processes respond to a history of errors. 


\section{Keywords}

human; motor learning; two-state model; error sensitivity; savings

60

63 We investigated what aspects of prior experience modulate error sensitivity, within the

64 framework of a two-state model of short-term sensorimotor adaptation. We manipulated initial

65 training on a visuomotor adaptation reaching task using specific perturbation schedules that are

66 thought to differentially affect fast and slow learning processes, and we tested what effect these

67 had on subsequent adaptation. We found that sensitivity to adaptation error was similarly

68 modulated by abrupt, gradual, and random perturbation schedules.

69

70

71

72

73

74 


\section{Introduction}

77 Adaptation is often defined as an error-driven process, in which the error experienced during a movement leads to a corrective adjustment in the motor output on the following movement (1-5).

79 Behavioural measures of adaptation are well characterized by state-space models $(1,4)$, which

80 represent trial-to-trial changes in movement as a function of how an error on a given trial affects

81 motor output on the subsequent trial. The update from one trial to the next, or the change in

82 motor output, is based on two parameters: a retention parameter which determines what

83 proportion of motor output is retained from trial to trial, and an error sensitivity parameter which

84 governs the proportion of error experienced on the current trial that is corrected for on the

85 subsequent trial.

86 Variations of the state-space model are built on the assumption that adaptation is the

87 product of multiple underlying processes with distinct timescales (3, 6-8). Researchers have

88 begun to provide neural evidence to strengthen the theory that sensorimotor learning is supported

89 by multiple processes $(9,10)$. An influential two-state model of short-term motor adaptation was

90 proposed by Smith et al. (3) that proposed a fast process that learns quickly but has poor

91 retention and a slow process that learns more slowly, but has strong retention.

92 The prevailing success of the two-state model continues to be that it accounts for the

93 learning phenomenon known as savings, characterized as prior learning speeding up subsequent

94 relearning $(3,11)$. While Smith et al. (3) initially argued that the reason for the fast relearning

95 during a second introduction of the same perturbation was due to the resistance of the slow

96 process to change, recent studies suggest that learning rate can be modified depending on factors

97 such as the uncertainty of movement error $(12,13)$, size of movement error (14), and a history of

98 movement errors (15-17). 
The behavioural changes associated with savings suggest that some component of

100 memory from the initial training must lead to the faster relearning, but what is remembered and

101 recalled remains unclear (15-22). One perspective argues for the enhancement of an explicit

102 strategy $(18,20,23)$, while the other side suggests that faster relearning is driven by the

103 experience of the motor errors $(15-17,24)$.

104 In support of the latter possibility, Herzfeld and colleagues (16) proposed that a history of

105 errors modulates the error sensitivity on each trial, systematically controlling how much the

106 motor system learns from the current motor error. They suggested that an error-based adaptation

107 model that provides for experience-dependent error sensitivity modification could account for

108 savings. Furthermore, Leow et al. (17) demonstrated that it is a memory of errors, not previous

109 actions, that is necessary for savings.

110 Recent work has shown that repeated exposure to the same force field perturbation results

111 in greater error sensitivity of both the fast and slow processes (15). While in Coltman et al. (15),

112 the error sensitivity terms for the fast and slow processes were held constant within a session, we

113 evaluated the theory of experience-dependent error sensitivity modulation in the context of

114 changes in error sensitivity from one learning session to the next. Although these results clearly

115 indicate that the motor system stored some component (i.e., memory) of prior training to speed

116 up subsequent learning, it remains unclear how the fast and slow learning processes contribute to

117 savings and what aspects of prior experience modulate error sensitivity. In other words, do both

118 fast and slow processes access a single stored component of prior training, or do they store

119 independent components? If the fast and slow processes depend on separate stored components

120 of prior training, then it may be possible to independently modulate characteristics of the fast and

121 slow processes (e.g., error sensitivity) by experimentally manipulating aspects of prior learning. 
Recent findings demonstrate that participants do not adapt linearly in response to

123 different magnitudes of error $(13,14,25)$. Additionally, Orban de Xivry and Lefevre (26)

124 propose that different perturbation schedules lead to distinct motor memories with different

125 attributes and neural representations (i.e., the amount of reorganization of the motor cortex). We

126 propose that perturbation schedules that are designed to produce learning using errors of

127 different magnitudes may have a differential effect on session-to-session changes to the fast

128 versus slow processes. In the present study, we manipulated initial training in a visuomotor

129 adaptation task using perturbation schedules which involved errors of different magnitudes, and

130 we tested what effect these different initial learning experiences had on subsequent adaptation,

131 and specifically on characteristics of the fast versus slow adaptation processes.

132 We asked one group of participants to counter a gradual perturbation schedule during

133 initial training. When a perturbation is gradually introduced, such that participants never

134 experience large errors, learning is believed to be more implicit in nature (26). We predicted that

135 when participants in this group were later tested on an abrupt perturbation, only the slow process

136 would be affected by the initial training, compared to a control group who were initially trained

137 using an abrupt perturbation. For a second group of participants, initial training was based on a

138 structural learning paradigm, involving a series of brief exposures to large, random perturbations

$139(27,28)$. This perturbation schedule is thought to be based on explicit learning mechanisms $(20$,

140 29). During the original conception of the two-state model, Smith et al. (3) designed a rebound

141 paradigm to test the theorized characteristics of each process. Much like the brief reversal used in

142 the rebound paradigm, which includes large errors and limited exposure, the motor output during

143 this phase of the paradigm is believed to be dependent on the fast process (3). For this group we 
144 predicted that when later tested on an abrupt perturbation, only the fast process would be affected

145 by the initial training, as compared to the control group.

146 We modelled perturbation-driven changes in movement with the state-space equations

147 proposed by Smith et al. (3), and focused on changes in the retention and error sensitivity

148 parameters. The model estimates function as a tool for understanding how the underlying

149 processes of adaptation were affected by the prior training. Substantiating the finding of Coltman

150 et al. (15), we confirm that repetition of the same visuomotor perturbation results in an increase

151 in error sensitivity for both processes, when comparing the two sessions of the control group. By

152 comparing the model estimates of participants in the gradual and structural learning groups to the

153 first session of the control, we expected to see changes in error sensitivity that depended on the

154 type of prior training participants experienced. Interestingly, however, we found that error

155 sensitivity of both the fast and slow processes was increased for both groups. The findings are

156 discussed in the context of storing and accessing a history of errors.

\section{Methods}

159 Participants. A total of 60 healthy young adults (age range 21-35; mean age \pm sd $27.9 \pm 4.2$

160 years) participated in a visuomotor rotation experiment. Participants were recruited from the

161 online platform maintained by Prolific.co and received $£ 11.25$ for their participation. As part of

162 the Prolific platform, participants respond to a series of questions related to age, gender, health

163 and economic status. Based on this prescreen information, 24 participants identified as female

164 and 36 as male. Participants were recruited globally and reported being located in 17 different

165 countries (Estonia, Finland, France, Greece, Hungary, Israel, Italy, Mexico, Netherlands, Poland,

166 Portugal, Slovenia, South Africa, Spain, Sweden, United Kingdom and the United States). All 
167 participants self-reported being right-handed and had normal or corrected-to-normal vision. The

168 protocol was approved by Western University's Research Ethics Board and all participants

169 indicated electronic consent.

170 Apparatus. Participants used a standard computer mouse and their own computer to access a

171 webpage hosted on a network computer located at Western Interdisciplinary Research Building.

172 The task was written in and controlled by JavaScript, running locally within the participants'

173 web browser.

Participants were asked to use a standard computer mouse and a standard credit or debit

175 card to complete a spatial calibration procedure. Participants were initially instructed how to turn

176 off the acceleration for the mouse, based on their operating system. Then, following an

177 instruction video, participants were asked to align the top of their mouse with the top of the

178 credit card. After a tone, they were instructed to move the mouse in a smooth and straight path,

179 aligning the top of their mouse with the bottom of the card. Participants were asked to hold still

180 while waiting for a second tone, indicating that they needed to realign the mouse with the top of

181 the card. This was repeated at two different speeds indicated in the video. When the calibration

182 procedure was successfully completed, participants watched an instructional video about the

183 experimental task.

184 The size and position of the stimuli were scaled based on a mouse calibration procedure.

185 Real-time position of the mouse was used to control the visual display and to provide on-line

186 visual feedback. The mouse speed was adjusted such that the distance from start position to

187 target was exactly $6 \mathrm{~cm}$ based on the calibration. While the physical target distance was always 6

$188 \mathrm{~cm}$, this translated to 300 pixels on screen. Therefore, the straight reach trajectory was 300

189 pixels, however a participant's view of this was potentially compressed or expanded relative to 
190 the target value of $6 \mathrm{~cm}$, depending on their monitor as well their viewing distance from the

191 monitor.

192 Paradigm. At the start of each trial, participants were instructed to click their mouse to begin. A

193 circular cursor (10 pixels radius) was virtually displayed on the participant's computer monitor

194 and was used to represent the position of the mouse on screen. The position of the mouse at the

195 start of the trial, represented the start position on screen. A small square (20 pixels by 20 pixels)

196 represented the target. The radial distance of the target from the start position was 300 pixels.

197 The target appeared at either $45^{\circ}, 90^{\circ}$, or $135^{\circ}$, relative to the start position (where $6 \mathrm{~cm}$ directly

198 to the right of the start position represented $0^{\circ}$ ). The location of the target was randomized per

199 trial, per participant, such that each participant saw a different order of targets with an equal

200 number of presentations of each target over the course of a session.

201 Participants were instructed to make a straight movement from the start position to the

202 target, within a narrow temporal window. At the beginning of each trial the target appeared in

203 white. Participants were required to hold still at the start position for $500 \mathrm{~ms}$, at which time the

204 target changed color to green, representing a "go" signal for participants to initiate a movement

205 to the target. In addition to the colour change of the target, a tone was used as a secondary "go"

206 signal. Participants needed to reach for the target and bring the centre of a red cursor

207 representing the position of their computer mouse within 10 pixels of the centre of the target

208 within 600-900 ms. If a participant's movement time was less than $600 \mathrm{~ms}$, the target turned red

209 to indicate that the movement was "too fast". If the participant's movement time was within

$210600-900 \mathrm{~ms}$, the target remained green to indicate that the movement was "good". If the

211 participant's movement time was greater than $900 \mathrm{~ms}$, the target would turn blue to indicate "too

212 slow". Feedback related to movement time was displayed on the screen for $1000 \mathrm{~ms}$ before the 
213 screen went blank and written instructions on screen indicated that the participant should return

214 the mouse to a comfortable starting position within their workspace. Participants were instructed

215 to try to obtain the "good" feedback as often as possible throughout the experiment.

216 To assist with making straight movements between the start position and the target using

217 a computer mouse, the first 20 trials of the first session represented a practice session for

218 participants. In these trials, a purple rectangle (50 pixels by 300 pixels), with two white lines on

219 either side was shown on screen, highlighting a straight path to the target. Participants were

220 instructed to keep the red cursor on the path, between the lines, toward the target. If the cursor

221 moved outside the path, the background colour changed from black to pink.

223 sessions (initial training and testing), separated by a 5-minute break (Fig. 1).

224 Each session included a total of 450 reaching movements, with a 1-minute mid-session break

225 halfway. The experimental paradigm for each session consisted of 4 epochs. The first epoch

226 (baseline) consisted of 70 trials in which participants were provided with veridical feedback of

227 the cursor position. The second epoch (adaptation) consisted of 300 trials in which a visuomotor

228 rotation was applied to the cursor feedback: an angular rotation was imposed on the cursor, such

229 that a hand movement aimed directly at a target produced a cursor movement that was rotated

230 radially about the start position and participants saw that their movement had generated an error.

231 Participants had to learn to counter the rotation by moving their hand in an equal and opposite

232 direction. With practice, participants adjusted their movements in such a manner that the visual

233 feedback produced straight trajectories from start position to the target. In the third epoch (error-

234 clamp; consisting of 30 trials) the task error was clamped to zero. During the clamp trials, the

235 angular position of the cursor relative to the start position was clamped to a straight line 
connecting the start position to the target, while participants maintained control of the radial

237 distance of the cursor from the start position. Finally, in the fourth epoch (washout; consisting of

23850 trials), participants were provided again with veridical feedback to bring performance back to

239 baseline.

During the adaptation epoch of the first session, participants experienced one of three

241 conditions: (1) a control learning group $(n=20)$ experienced an abrupt $30^{\circ}$ clockwise (CW)

242 rotation for all 300 trials during this phase (Fig. 1; top), (2) a gradual learning group, $(n=20)$ in

243 which a rotation was increased linearly from $0^{\circ}$ to $30^{\circ} \mathrm{CW}$ over 250 trials and then held at a

244 fixed $30^{\circ} \mathrm{CW}$ for another 50 trials (Fig. 1; middle), or (3) a structural learning group (n=20) in

245 which participants encountered random rotations, ranging from $60^{\circ}$ counter-clockwise $(\mathrm{CCW})$ to

$24660^{\circ} \mathrm{CW}$ in blocks of 6 trials with the same rotation $(27,28,29$; Fig. 1; bottom). In this group, we

247 deliberately set the average over all angles to zero, to prevent any accumulative learning. We

248 also excluded rotation sizes within $10^{\circ}$ of the test rotation $\left(30^{\circ} \mathrm{CW}\right)$ and its inverse $\left(30^{\circ} \mathrm{CCW}\right)$.

249 We furthermore set the change in rotation angle to be equal to or greater than $15^{\circ}$ to ensure the

250 errors were always large, which characteristically has the greatest influence on the fast process

$251(3,29)$. During the second session, all three groups experienced an abrupt $30^{\circ} \mathrm{CW}$ rotation

252 during the adaptation epoch.

253 Data Analysis. The position of the cursor in both x (lateral) and y (sagittal), were sampled in

254 pixels at the refresh rate of their computer monitor (typically $60 \mathrm{~Hz}$ ). Missed samples were

255 interpolated during analysis (less than $1 \%$ of samples on average). In cases in which data were

256 acquired at higher sampling rates (for example because a participant's computer monitor refresh

257 rate exceeded $60 \mathrm{~Hz}$ ), the data were down sampled to $60 \mathrm{~Hz}$. Data were digitally smoothed using 
a second-order low-pass Butterworth filter with a cut-off frequency of $15 \mathrm{~Hz}$. All data were stored for offline analysis using custom MATLAB R2020a (The MathWorks) scripts. was defined as the time at which the tangential velocity of the mouse first exceeded $0.5 \mathrm{~cm} / \mathrm{s}$ and movement end was defined as the first time after peak velocity that tangential velocity fell below

$2630.5 \mathrm{~cm} / \mathrm{s}$, where peak velocity was defined as the fastest participants ever moved during the reach 264 movement. For each trial we computed the angle between the line connecting the start position 265 and the cursor position at peak velocity, and the line connecting the start position to the target.

266 We determined the average reach angle, per subject during the last 50 trials of the baseline epoch 267 and we subtracted this quantity from the reach angle measured on each trial.

268 Model fitting. Smith et al. (3) outlined a method for mathematically modelling an iterative 269 update of the states of the two proposed processes of short-term sensorimotor adaptation.

270 Essentially, the model involves fitting four parameters: an error sensitivity and a retention 271 parameter for both a fast and a slow process. The first parameter weighs the relative importance 272 of recalling previous motor commands, which is interpreted as the retention factor. The second 273 parameter is the sensitivity to error, which relates to the proportion of error that is corrected for 274 trial-to-trial $(1,3,4,30)$. The two important assumptions in this model are that the error 275 sensitivity is higher for the fast process compared with the slow process and that retention is 276 stronger for the slow process compared with the fast process (3). Adaptation can be decomposed 277 into a fast (Eq. 1) and a slow (Eq. 2) process, knowing that each state follows different learning 278 dynamics. The two processes are summed together to produce the overall output x (Eq. 3). Error, 279 denoted by e(n), arises on each trial $\mathrm{n}$ as the difference between the overall output xnet and the 280 task parameter r (i.e., the degree of the rotation; Eq.4). 


$$
\begin{gathered}
x_{f}(n+1)=A_{f} x(n)+B_{f} e(n) \\
x_{s}(n+1)=A_{s} x(n)+B_{s} e(n) \\
x_{n e t}(n)=x_{f}(n)+x_{s}(n) \\
e(n)=r(n)-x_{n e t}(n)
\end{gathered}
$$

dynamics (31):

$$
\begin{aligned}
& A_{f} \leq A_{s}+0.001 \\
& B_{f} \geq B_{s}+0.001
\end{aligned}
$$

In order to approximate the four parameters (i.e., Af, As, Bf, and Bs), we fit the model to

291 the behavioral data (using the function fmincon in MATLAB r2020a) by minimizing the squared

292 difference between the estimated net output (xnet) of the model and the average participant reach

293 angle, measured on each trial. According to the methods described in Albert and Shadmehr (31),

294 we also included a mathematical formalization of visual error clamp trials and set breaks.

295 Statistical Design. Pairwise comparisons were performed with nonparametric bootstrap

296 hypothesis tests, as well as paired and unpaired t-tests. For statistical analyses that require

297 multiple comparisons, we used the Holm-Bonferroni correction (32). Statistical tests were

298 considered significant at $\mathrm{p}<0.05$. For all reported and depicted values, we report the mean and

299 SEM.

\section{Results}

Figure 2 shows the hand paths from one representative participant in the control group

302 during both sessions one and two, as well as one representative participant per group in session

303 two of the structural and gradual learning groups. During the baseline epoch (left column), these

304 paths are relatively straight to the target. The representative participants were all adapting to an

305 abrupt $30^{\circ} \mathrm{CW}$ rotation. During the early adaptation epoch (middle column) these movements 
were initially deviated in the $\mathrm{CW}$ direction, with a corrective movement at the end of the trajectory to bring the cursor to the target. In all three groups, participants adapted to the $30^{\circ} \mathrm{CW}$ rotation by the late adaptation epoch (right column), reducing their movement errors and resuming relatively straight hand paths to the target.

311 outcome measure for the study was reach angle at peak velocity, which was measured as the

312 angle between the straight line connecting the start position and the cursor position at peak

313 velocity and the straight line connecting the start position to the target. The control group of

314 participants adapted their movements to an abrupt $30^{\circ} \mathrm{CW}$ visuomotor rotation in both the first

315 and second session. Figure 3A shows the angle at peak velocity for all trials in each session,

316 averaged across participants in the control group. In both sessions, participants exhibited learning

317 during the adaptation epoch, decay during the visual error clamp epoch, and a return towards

318 baseline performance during the washout epoch. During the adaptation epoch we examined the 319 learning at two different time points: early (first fifty trials during adaptation) and late (last fifty

320 trials during adaptation; Fig. 3B). The mean angle in the early learning phase of the second 321 session $(M=23.7, S D=3.15)$ was reliably greater than in the first session $[M=19.9, S D=$ 322 4.6; paired $t$-test, $t(19)=-6.2, P=3.0 \mathrm{e}-06]$, indicating savings. We did not detect a reliable 323 difference $(P=0.08)$ between sessions during late learning.

324 A second group of participants was exposed to a gradual perturbation schedule during

325 initial training. Figure 4A shows the angle at peak velocity for all trials in each session, averaged 326 across participants in the gradual learning group. Participants exhibited learning during the 327 adaptation epoch, decay during the visual error clamp epoch, and a return towards baseline 328 performance during the washout epoch. A final group of participants was exposed to a series of 
329 brief exposures to large, random perturbations. Each participant in this group experienced a 330 different set of randomly varying rotations. Figure 4B illustrates the angle at peak velocity for

331 all trials in session one for four representative individual participants from the structural learning

332 group. We observed two participants who demonstrated learning within each block of six trials,

333 but who also appeared to have maintained a fraction of error throughout the adaptation epoch

334 (Fig. 4B, S2 and S8). In addition to a participant who adapted quickly to the randomly changing

335 perturbation (Fig. 4B, S18), we observed a participant who qualitatively showed greater

336 reduction of error in the latter half of the adaptation epoch, compared to the early half (Fig. 4B,

337 S20).

Figure 5A shows the angle at peak velocity averaged across participants for all trials in

339 session one of the control group and session two of the structural and gradual learning groups.

340 When comparing the model estimates of participants in the gradual and structural learning

341 groups during the second session to the first session of the control group, we expected to see

342 changes in error sensitivity that depended on the type of prior training participants experienced.

343 To compare the changes in angle between the control, structural and gradual learning groups, we

344 examined learning during the adaptation epoch at two different time points: early (first fifty trials

345 during adaptation) and late (last fifty trials during adaptation; Fig. 5B). A one-way ANOVA

346 revealed a significant effect of mean angle between the control, structural and gradual learning

347 groups during early learning $[F(2,57)=14.4, P=8.8$ e-06].

348 Post hoc comparisons using Tukey HSD tests indicated that the mean angle for the

349 structural learning group $(M=24.9, S D=2.9, P=2.9$ e- 05$)$ and the gradual learning group $(M=$

$35024.5, S D=2.9, P=1.4$ e-04) were reliably higher than the mean angle for the control group ( $M$

$351=19.9, S D=4.6)$. However, there was no reliable difference detected between the structural and 
352 gradual learning groups $(P=0.9)$. During late learning, we did not detect a reliable difference in

353 mean angle among the groups $(P=0.2)$. Therefore, the structural and gradual learning groups

354 demonstrated fast learning when countering an abrupt $30^{\circ} \mathrm{CW}$ rotation, as compared to session

355 one of the control group. While the control group represented naive learners, the prior experience

356 from session one for the structural and gradual learning groups is suggested to have facilitated

357 the improved learning. Likewise, this was observed in the control group, in which participants

358 experienced a repetition of an abrupt rotation and demonstrated savings during the second

359 session.

360 Recent work suggests that error sensitivity in sensorimotor adaptation is likely not

361 constant, but rather can vary depending on prior experience $(13,14,16,33)$. We modelled

362 movement angle across each session with the state-space equations proposed by Smith et al. (3),

363 and focused on changes in the retention and error sensitivity parameters. The main objective of

364 this study was to compare the model parameters across groups learning to counter the abrupt $30^{\circ}$

365 CW rotation. To do this, we used the bootstrap procedure previously reported by Coltman et al.

366 (15). In this manner, we always fit the model to averaged group data for each resampled

367 population $(15,31)$. The estimated posterior distributions of each of the four two-state model

368 parameter values are depicted in Fig. 6 for sessions one and two of the control group and session

369 two of the gradual and structural learning groups. To determine whether the difference between

370 the mean of each distribution was statistically reliable, we calculated the distribution of the

371 differences in individual samples. The insets in Fig. 6 show the distribution of differences found.

372 Table 1 shows the mean and standard deviation for each of the two-state parameters for each

373 group. 
We first compared parameter estimates from session one and session two for the control group (Fig. 6A). Across all comparisons made between groups, we did not observe a reliable

376 difference in the retention parameters for either the fast or slow process. When participants

377 experienced repetition of the same abrupt rotation, we found a statistically reliable increase in the 378 error sensitivity parameter for both the fast $\left(\mathrm{B}_{f}, P=0.007\right)$ and the slow $\left(\mathrm{B}_{s}, P=0.003\right)$

379 processes. Importantly, this comparison allowed us to demonstrate that our previous finding from 380 a force field adaptation task (15) was replicated in a visuomotor rotation task. Therefore, this 381 result suggests that both the fast and slow processes are responsive to a history of error and both 382 contribute to savings.

383 Next, we compared parameter estimates from session one of the control group with

384 session two of the structural learning group (Fig. 6B). Based on the theory of structural learning, 385 thought to be essential to capturing the initial rapid phase of learning, Braun et al. (27)

386 demonstrated that the benefit of knowing the underlying structure of a task is that it leads to 387 facilitated adaptation. For this group we predicted that when later tested on an abrupt 388 perturbation, only the fast process would be affected by the initial training, as compared to the 389 control group. When overall learning is decomposed into a fast and slow state, the initial rapid 390 phase of learning is dominated by the output of the fast process. Therefore, we assumed that such 391 practice would influence the fast process. In addition to a statistically reliable increase in the 392 error sensitivity parameter for the fast process $\left(\mathrm{B}_{f}, P<0.001\right)$, we also found a statistically 393 reliable increase in the slow process error sensitivity $\left(\mathrm{B}_{s}, P=0.002\right)$.

394 Learning is believed to be more implicit in nature when a perturbation is gradually 395 applied using small undetectable increases, so that participants never encounter large sensory 396 prediction errors $(22,26,34)$. By exposing a group of participants to a gradual perturbation 
397 schedule during initial training, we predicted that only the slow process would be influenced.

398 When we compared the parameter estimates from session one of the control group with session

399 two of the gradual learning group (Fig. 6C) we found the gradual learning group showed a

400 statistically reliable increase in the error sensitivity parameter for both the fast $\left(\mathrm{B}_{f}, P=0.009\right)$

401 and the slow $\left(\mathrm{B}_{s}, P=2 \mathrm{e}-04\right)$ processes.

402 Lastly, we compared parameter estimates between the structural and gradual learning

403 groups during session two (Fig. 6D). Our goal was to use two different adaptation schedules

404 thought to differentially affect fast and slow learning processes and test the idea that error

405 sensitivity for each process would be independently modulated. We expected that the error

406 sensitivity parameter for the fast process would be greater in the structural learning group

407 compared to the gradual learning group, while the error sensitivity parameter for the slow

408 process would be greater in the gradual learning group compared to the structural learning group.

409 The only statistically reliable difference was in the error sensitivity parameter for the fast process

410 that was larger for the structural learning group $\left(\mathrm{B}_{f}, P=0.02\right)$.

411 From the bootstrap distributions we calculated the mean value for each parameter for

412 session one and session two of the control group, and session two of the structural and gradual

413 learning groups separately. Using these mean estimated parameter values, we used the two-state

414 model to simulate our experimental paradigm and generate simulated learning curves to visualize

415 the time course of the estimated fast and slow processes, as well as the simulated overall output.

416 Figure 7 demonstrates that the simulated learning curves are qualitatively in good agreement

417 with the measured behavioural data. The models explains $98-99 \%$ of the variance in angle over

418 the course of learning (control session $1: \mathrm{R}^{2}=0.98, P=2.2 \mathrm{e}-04$; control session $2: \mathrm{R}^{2}=0.99, P$

$419=1.2 \mathrm{e}-04 ;$ structural session $2: \mathrm{R}^{2}=0.98, P=2.3 \mathrm{e}-04$; gradual session $2: \mathrm{R}^{2}=0.99, P=1.6 \mathrm{e}-$ 
42004 ). The model effectively captures the initial improvement in learning during the adaptation

421 epoch, the decay during the visual error clamp epoch, as well as the subsequent return towards

422 baseline performance during the washout epoch.

\section{Discussion}

424 The integration of different perturbation schedules and two-state modelling of measured

425 behavioral data allowed us to test the role of prior experience on error sensitivity modulation

426 during subsequent adaptation. The modelling of the data in turn describes adaptation as an

427 interaction between error-sensitivity and retention. It has previously been shown in the context of

428 force field learning that repetition of the same perturbation results in increased error sensitivity

429 for both the fast and slow processes of adaptation (15). We substantiated this here by

430 demonstrating that sensitivity to errors is similarly increased for both the fast and slow processes

431 during the second session of a visuomotor rotation task. We found no reliable differences in the

432 retention parameter across conditions and sessions.

433 The behavioural changes associated with savings suggest that some component of

434 memory from the initial training must lead to the faster relearning, but what is remembered and

435 recalled remains unclear. In the context of the present study, how the fast and slow processes

436 individually contribute to savings, is not well known. To address this point, we used different

437 perturbation schedules that relied on errors of different magnitudes to determine whether the

438 underlying processes of adaptation could be independently manipulated, and whether an

439 independent memory would subsequently be formed. We expected to see differences in error

440 sensitivity depending on the type of prior training participants had received and therefore

441 compared the model parameter estimates of participants in the gradual and structural learning

442 groups to the first session of the control group, but we found that error sensitivity of both the fast 
443 and slow processes was increased for both groups. Such a result might suggest that sensitivity to

444 error during visuomotor adaptation is modulated by abrupt, gradual and random perturbation

445 schedules.

446 As an alternative account, savings has previously been explained by the retrieval of

447 previous successful actions, reflecting the use of an explicit strategy $(18-20,23)$. Within the

448 framework of a two-state model, this theory suggests that savings is driven purely by the fast

449 process, without consideration of the contributions from the slow process (35). Several

450 researchers have argued that explicit cognitive strategies can account for a significant amount of

451 learning, particularly during the early phase of learning and relearning (36-38). The dissociation

452 of learning into implicit and explicit learning processes often relies on the use of verbal aiming

453 reports prior to reaching $(18,20,23,38)$. Recent findings, however, indicate that verbal aiming

454 reports could lead to an overestimated explicit contribution to adaptation $(21,39)$. In fact, Leow

455 et al. (21) demonstrated that the use of shortened preparation time, designed to prevent strategic

456 re-aiming, resulted in the estimated implicit learning being larger than that which was obtained

457 from verbal reports. Furthermore, Yin and Wei (22) provide supporting evidence that savings of

458 motor adaptation is possible without forming or recalling a cognitive strategy with the use of a

459 gradually introduced visuomotor rotation during initial learning. If savings is possible, with and

460 without an explicit strategy being formed during initial learning and predominant measures of

461 implicit and explicit processes may be confounding their mode of measurement, how reliable are

462 the findings suggesting savings is driven exclusively by an explicit process?

463 The debate about the contributions of explicit/implicit and fast/slow processes to savings

464 stems from a recent proposal that fast and slow processes reflect explicit and implicit learning

465 mechanisms, respectively (35). Motor memory development is thought to be based on two 
components: recall, which involves retrieving past motor movements, and faster relearning,

467 which involves increased sensitivity to errors $(3,40)$. By using the framework of a two-state

468 model we focused on the dominant component of adaptation, which is sensory prediction error,

469 and found that motor memory was associated with an increase in error sensitivity. In addition to

470 sensory prediction error, there are other possible teaching cues that might drive adaptation. When

471 researchers are focused on the more cognitive aspects of learning, exploring the use of an explicit

472 strategy, the dominant component of adaptation may likely be the reinforcement of successful

473 actions. Future research may shed light on this debate by probing both proposed methods of dual

474 processing simultaneously during learning, and by assessing more directly their shared features.

475 Another long-standing question is how quickly implicit changes in learning emerge.

476 Huberdeau and colleagues (20) demonstrated that learning of an abrupt perturbation with only a

477 few trials is sufficient to cause savings via the explicit process, based on the belief that the fast

478 learning is too short for implicit learning to take its full effect. Ruttle et al. (41) however recently

479 confronted the long standing notion that implicit learning is slowly developing, typically

480 unfolding over tens of trials. By observing changes in both internal models and state estimates of

481 limb position as a characterization of implicit learning, they found that after only one to three

482 perturbed training trials participants had changes in both reach aftereffects and a shift in hand

483 localization. Taking this into account, it seems possible that the 6-trial repetition used in the

484 structural learning task, aiming to influence the fast process, may have simultaneously influenced

485 the slow process. For that reason, it is possible that a common component of all three

486 perturbation schedules used during initial training was that the slow process accounted for a

487 significant portion of the error reduction. 
Albert et al. (33) recently investigated the persistence of residual errors during motor adaptation in the context of implicit and explicit learning systems. Of importance to the present study, they propose that it is the implicit learning system which maintains a history for prior

491 errors. Our results are consistent with this hypothesis that it is the implicit process that stored 492 some component of prior training. Given the suggestion that the history of errors is stored by

493 only one of the two proposed underlying processes, this finding would be lost if learning

494 behavior was represented using a single-state model. Nevertheless, one may ask whether a two-

495 state model was necessary to represent learning in the behavioral tasks tested in the present

496 study. To address this, we calculated AIC values for both single- and two-state models fits to the 497 behavioral data. We used the data associated with the four sessions of abrupt rotations (i.e., the

498 first and second sessions of the control group, and the second session of the gradual and 499 structural learning groups) and for each we estimated the overall output based on a single-state 500 model and separately using a two-state model. Based on the model with the lowest AIC value, in 501 all four cases the best-fit model describing the measured behavioural data was the two-state 502 model. As a follow-up to our initial question, we would further suggest that the stored memory is 503 accessible to both processes during subsequent learning. As it pertains to our findings, we would 504 argue that while the fast process may not maintain a history or errors, it does have access to this 505 information in subsequent learning as evident by the increased error-sensitivity for the fast 506 process during testing in all groups.

Alternatively, while the experimental design and two-state model, used in the present 508 study, account well for the results of savings, recent work looking at evoked recovery (42) posits 509 that memory formation is related to the storing of information about the dynamical and sensory 510 features of the environment is related to the context with which it is associated. Understanding 
bioRxiv preprint doi: https://doi.org/10.1101/2021.06.14.448375; this version posted July 18, 2021. The copyright holder for this preprint (which was not certified by peer review) is the author/funder, who has granted bioRxiv a license to display the preprint in perpetuity. It is made available under aCC-BY 4.0 International license.

511 how contextual inference can be related to and accessed by each process of the two-state model

512 can shed light on future discussions about multiple processes underlying motor learning. 
bioRxiv preprint doi: https://doi.org/10.1101/2021.06.14.448375; this version posted July 18,2021 . The copyright holder for this preprint (which

was not certified by peer review) is the author/funder, who has granted bioRxiv a license to display the preprint in perpetuity. It is made available under aCC-BY 4.0 International license.

514

Table 1. Two-state model parameters calculated from probability distribution

\begin{tabular}{llllc}
\hline & \multicolumn{2}{c}{ Fast Process } & \multicolumn{2}{c}{ Slow Process } \\
\cline { 2 - 5 } & $A($ mean $\pm \mathrm{SD})$ & $B($ mean $\pm \mathrm{SD})$ & $A($ mean $\pm \mathrm{SD})$ & $B($ mean $\pm \mathrm{SD})$ \\
\hline Control Session 1 & $0.86(0.02)$ & $0.17(0.02)$ & $0.996(7 \mathrm{e}-04)$ & $0.05(0.006)$ \\
\hline Control Session 2 & $0.87(0.03)$ & $0.28(0.04)$ & $0.994(0.001)$ & $0.07(0.01)$ \\
\hline Structural Session 2 & $0.80(0.05)$ & $0.40(0.03)$ & $0.993(0.002)$ & $0.10(0.02)$ \\
\hline Gradual Session 2 & $0.87(0.02)$ & $0.27(0.04)$ & $0.994(0.001)$ & $0.10(0.02)$ \\
\hline
\end{tabular}




\section{Figure Captions}

Figure 1. Experimental design and perturbation schedule. The experiment was divided into two sessions, separated by a 5-min session break. Each session consisted of four blocks: 1) a baseline period of no rotation trials, 2) an adaptation period, 3) an error clamp period, and 4) a washout period. Participants were randomly assigned to one of three groups which differed in session one during the adaptation period: abrupt control group, gradual learning group, or structural learning group.

Figure 2. Hand trajectories from a representative participant in the control group during both session one (light blue) and two (dark blue), and one representative participant per group in session two of the structural (purple) and gradual (pink) learning groups. Baseline reaches were from the last three trials (from trial 68 to trial 70) during the baseline epoch. Early and late adaptation reaches were from the first (from trial 71 to 73 ) and last (from trial 368 to trial 370) three trials of the adaptation epoch, respectively. Participants saw a random ordering of the three possible targets (represented by the squares).

Figure 3. Control group. $A$ : the average angle at peak velocity for all trials in session 1 (light blue) and session 2 (dark blue). The shaded region denotes \pm SE. $B$ : comparisons between session 1 and session 2 (dark blue) for the mean angle for the first 50 (early) and last 50 (late) trials of the adaptation epoch. Circles represent individual data.

Figure 4. Gradual and structural learning groups. $A$ : the average angle at peak velocity for all trials in session 1 of the gradual learning group. The shaded region denotes $\pm \mathrm{SE}$. $B$ : the data from four representative individual participants (S2, top left, S8 top right, S18 bottom left, S20 bottom right).

Figure 5. $A$ : the average angle at peak velocity for all trials in session 1 for the control group (light blue) and session 2 for the structural (purple) and gradual (pink) learning groups. The shaded region denotes $\pm \mathrm{SE}$. $B$ : comparisons between groups for the mean angle for the first fifty (early) and last 50 (late) trials of the adaptation epoch. Circles represent individual data.

Figure 6. Probability distribution of the model parameters given the data. Light blue and dark blue represent session 1 and session 2 of the control group, respectively. Purple represents session 2 of the structural learning group and Pink represents session 2 of the gradual learning group. Inset represents the distribution of pairwise differences. The four model parameters of the two-state model are fast retention $\left(A_{f}\right)$, slow retention $\left(A_{s}\right)$, fast learning rate $\left(B_{f}\right)$, and slow learning rate $\left(\mathrm{B}_{\mathrm{s}}\right)$.

Figure 7. Model simulations. Parameter estimates for each session were based on the mean values from the bootstrap distributions shown in Fig. 6. The four parameters of the model are fast retention $(\mathrm{Af})$, fast learning rate $(\mathrm{Bf})$, slow retention $(\mathrm{As})$, and slow learning rate $(\mathrm{Bs})$. 
563 1. Donchin O, Francis JT, and Shadmehr R. Quantifying generalization from trial-by-

564 trial behavior of adaptive systems that learn with basis functions: theory and experiments in

565 human motor control. Journal of Neuroscience 23: 9032-9045, 2003.

5662 2. Miall RC, and Wolpert DM. Forward models for physiological motor control. Neural 567 networks 9: 1265-1279, 1996.

568 3. Smith MA, Ghazizadeh A, and Shadmehr R. Interacting adaptive processes with different timescales underlie short-term motor learning. PLoS Biol 4: e179, 2006. of motor primitives. Nature 407: 742-747, 2000.

5725 Wolpert DM, and Kawato M. Multiple paired forward and inverse models for motor

573 control. Neural networks 11: 1317-1329, 1998.

574 6. Kording KP, Tenenbaum JB, and Shadmehr R. The dynamics of memory as a consequence of optimal adaptation to a changing body. Nature neuroscience 10: 779-786, 2007. memory. Journal of Neuroscience 29: 10396-10404, 2009.

$578 \quad 8 . \quad$ Coltman SK, and Gribble PL. Time course of changes in the long-latency feedback response parallels the fast process of short-term motor adaptation. J Neurophysiol 124: 388-399, 5802020.

$581 \quad$ 9. Kim S, Ogawa K, Lv J, Schweighofer N, and Imamizu H. Neural substrates related to motor memory with multiple timescales in sensorimotor adaptation. PLoS biology 13: e1002312, 2015.

584 10. Sarwary AM, Wischnewski M, Schutter DJ, Selen LP, and Medendorp WP.

585 Corticospinal correlates of fast and slow adaptive processes in motor learning. Journal of 586 neurophysiology 120: 2011-2019, 2018.

587 11. Ebbinghaus H. Memory: A Contribution to Experimental Psychology, eds. HA Ruger and CE Bussenius (Trans)(Dover Publications, New York, 1964)(Original Work Published 1885) 1913.

590 12. van Beers RJ. Motor learning is optimally tuned to the properties of motor noise. Neuron 591 63: 406-417, 2009.

592 13. Wei K, and Kording K. Relevance of error: what drives motor adaptation? Journal of 593 neurophysiology 101: 655-664, 2009.

594 14. Marko MK, Haith AM, Harran MD, and Shadmehr R. Sensitivity to prediction error in reach adaptation. Journal of neurophysiology 108: 1752-1763, 2012.

596 15. Coltman SK, Cashaback JG, and Gribble PL. Both fast and slow learning processes 597 contribute to savings following sensorimotor adaptation. Journal of neurophysiology 121: 1575$598 \quad 1583,2019$.

599 16. Herzfeld DJ, Vaswani PA, Marko MK, and Shadmehr R. A memory of errors in 600 sensorimotor learning. Science 345: 1349-1353, 2014.

601 17. Leow L-A, De Rugy A, Marinovic W, Riek S, and Carroll TJ. Savings for visuomotor 602 adaptation require prior history of error, not prior repetition of successful actions. Journal of 603 neurophysiology 116: 1603-1614, 2016.

604 18. Avraham G, Morehead JR, Kim HE, and Ivry RB. Reexposure to a sensorimotor 605 perturbation produces opposite effects on explicit and implicit learning processes. PLoS biology 606 19: e3001147, 2021. 
19. Huang VS, Haith A, Mazzoni P, and Krakauer JW. Rethinking motor learning and savings in adaptation paradigms: model-free memory for successful actions combines with internal models. Neuron 70: 787-801, 2011. 20. Huberdeau DM, Haith AM, and Krakauer JW. Formation of a long-term memory for visuomotor adaptation following only a few trials of practice. Journal of neurophysiology 114: 969-977, 2015. of visuomotor rotation learning by constraining movement preparation time. Journal of neurophysiology 118: 666-676, 2017.

616 22. Yin C, and Wei K. Savings in sensorimotor adaptation without an explicit strategy.

617 Journal of neurophysiology 123: 1180-1192, 2020.

618 23. Morehead JR, Qasim SE, Crossley MJ, and Ivry R. Savings upon re-aiming in

619 visuomotor adaptation. Journal of neuroscience 35: 14386-14396, 2015.

$620 \quad 24 . \quad$ Hanajima R, Shadmehr R, Ohminami S, Tsutsumi R, Shirota Y, Shimizu T, Tanaka

621 N, Terao Y, Tsuji S, and Ugawa Y. Modulation of error-sensitivity during a prism adaptation

622 task in people with cerebellar degeneration. Journal of neurophysiology 114: 2460-2471, 2015.

623 25. Kim HE, Morehead JR, Parvin DE, Moazzezi R, and Ivry RB. Invariant errors reveal

624 limitations in motor correction rather than constraints on error sensitivity. Communications

625 Biology 1: 1-7, 2018.

626 26. Orban de Xivry J-J, and Lefèvre P. Formation of model-free motor memories during

627 motor adaptation depends on perturbation schedule. Journal of neurophysiology 113: 2733-2741,

6282015.

629 27. Braun DA, Aertsen A, Wolpert DM, and Mehring C. Motor task variation induces

630 structural learning. Current Biology 19: 352-357, 2009.

631 28. Braun DA, Mehring C, and Wolpert DM. Structure learning in action. Behavioural

632 brain research 206: 157-165, 2010.

633 29. Bond KM, and Taylor JA. Structural learning in a visuomotor adaptation task is

634 explicitly accessible. Eneuro 4: 2017.

635 30. Scheidt RA, Dingwell JB, and Mussa-Ivaldi FA. Learning to move amid uncertainty.

636 Journal of neurophysiology 86: 971-985, 2001.

637 31. Albert ST, and Shadmehr R. Estimating properties of the fast and slow adaptive

638 processes during sensorimotor adaptation. Journal of Neurophysiology 119: 1367-1393, 2018.

639 32. Holm S. A simple sequentially rejective multiple test procedure. Scandinavian journal of 640 statistics 65-70, 1979.

$64133 . \quad$ Albert ST, Jang J, Sheahan HR, Teunissen L, Vandevoorde K, Herzfeld DJ, and

642 Shadmehr R. An implicit memory of errors limits human sensorimotor adaptation. Nature

643 Human Behaviour 2021.

644 34. Criscimagna-Hemminger SE, Bastian AJ, and Shadmehr R. Size of error affects cerebellar contributions to motor learning. Journal of neurophysiology 103: 2275-2284, 2010. 35. McDougle SD, Bond KM, and Taylor JA. Explicit and implicit processes constitute the fast and slow processes of sensorimotor learning. Journal of Neuroscience 35: 9568-9579, 2015. 36. Mazzoni P, and Krakauer JW. An implicit plan overrides an explicit strategy during visuomotor adaptation. Journal of neuroscience 26: 3642-3645, 2006.

65037 . Taylor JA, and Ivry RB. Flexible cognitive strategies during motor learning. PLoS 651 computational biology 7: e1001096, 2011. 
652 38. Taylor JA, Krakauer JW, and Ivry RB. Explicit and implicit contributions to learning 653 in a sensorimotor adaptation task. Journal of Neuroscience 34: 3023-3032, 2014.

65439 . de Brouwer AJ, Albaghdadi M, Flanagan JR, and Gallivan JP. Using gaze behavior

655 to parcellate the explicit and implicit contributions to visuomotor learning. Journal of

656 neurophysiology 120: 1602-1615, 2018.

657 40. Mawase F, Bar-Haim S, and Shmuelof L. Formation of Long-term Locomotor

658 memories is associated with functional connectivity changes in the cerebellar-thalamic-cortical 659 network. Journal of Neuroscience 37: 349-361, 2017.

660 41. Ruttle JE, Marius't Hart B, and Henriques DY. Implicit motor learning within three 661 trials. Scientific Reports 11: 1-11, 2021.

662 42. Heald J, Lengyel M, and Wolpert D. Contextual inference underlies the learning of 663 sensorimotor repertoires. bioRxiv 2020.

664 


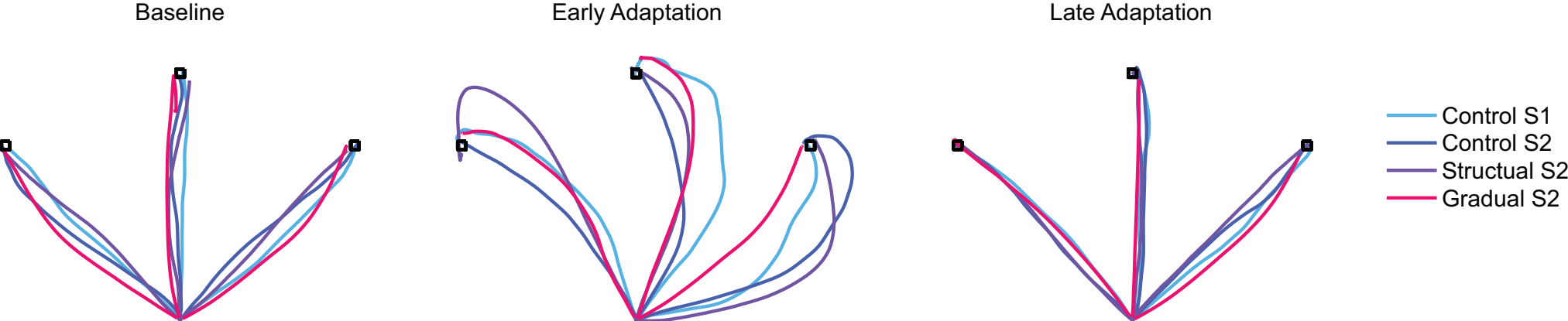



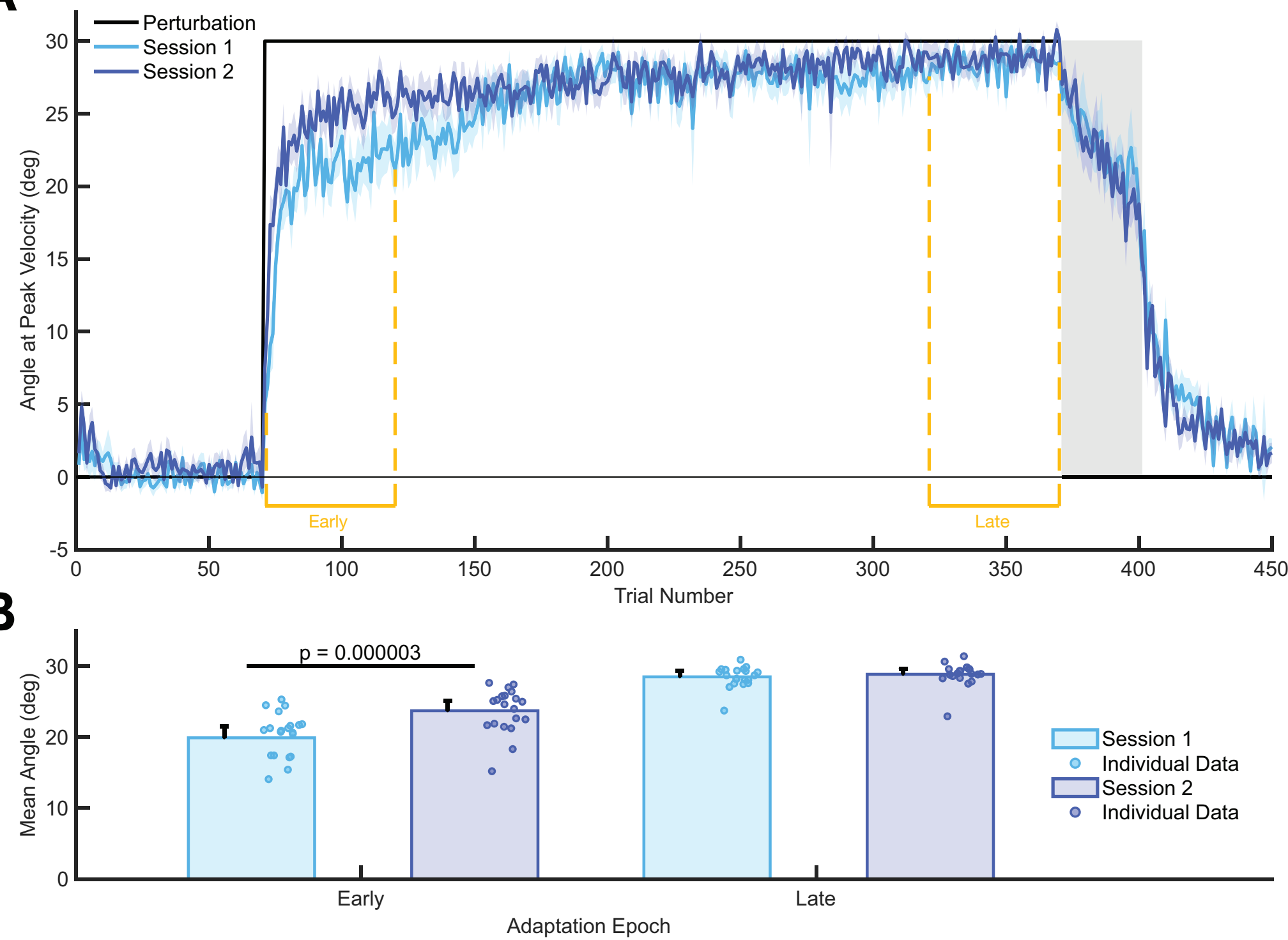
A

B
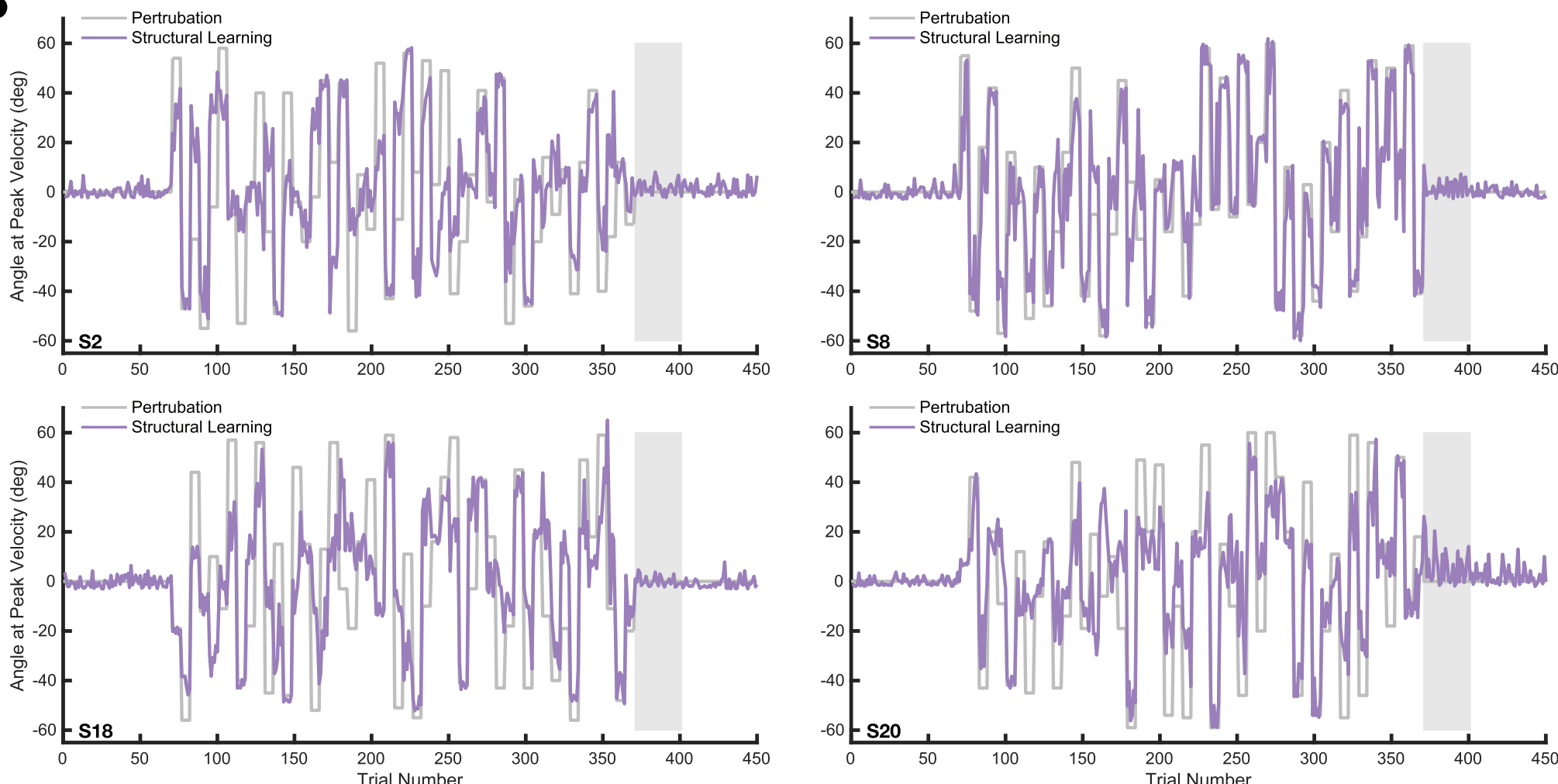

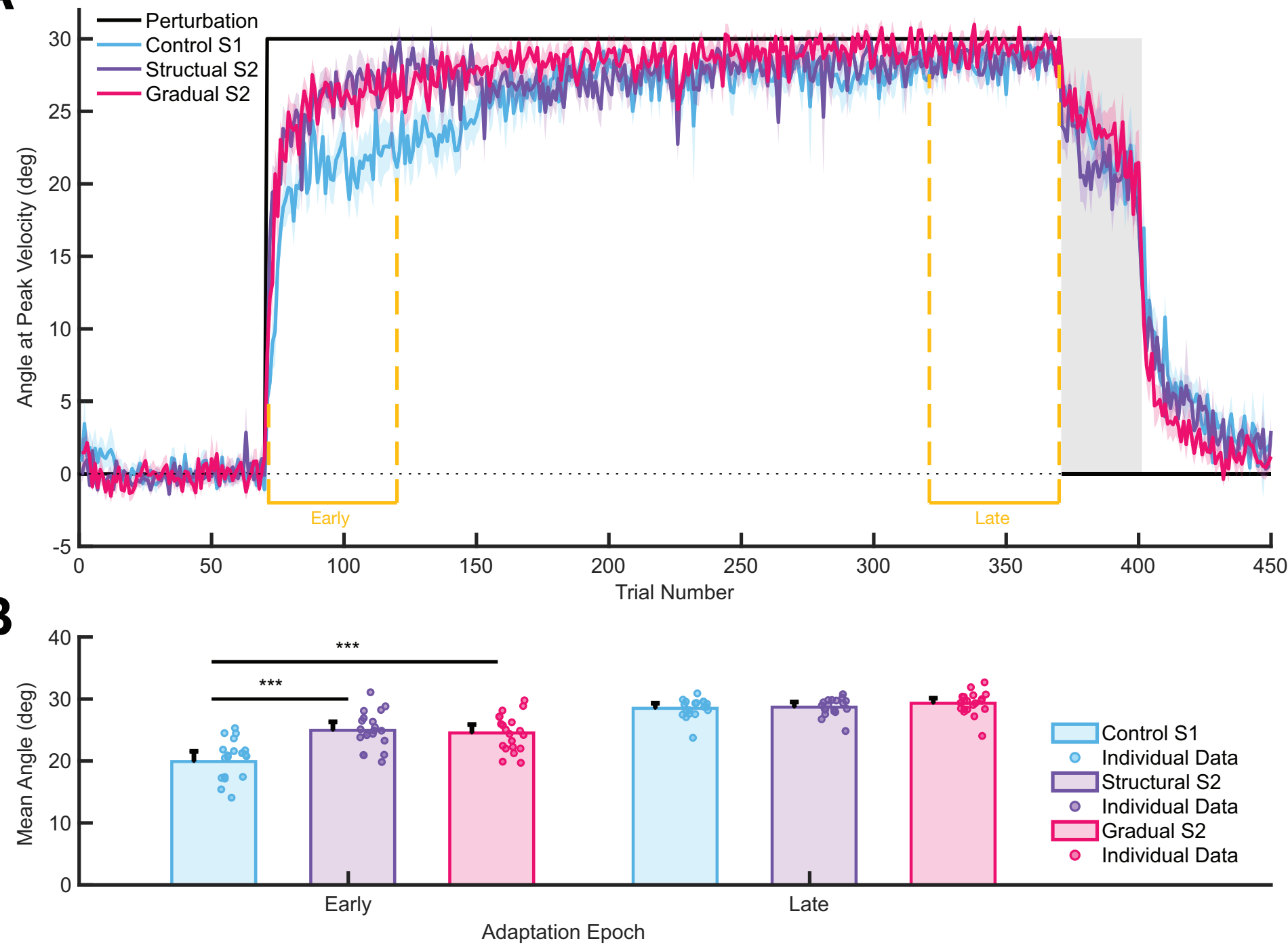
A

\section{Control Session 1 vs} Control Session 2

Fast Retention Af
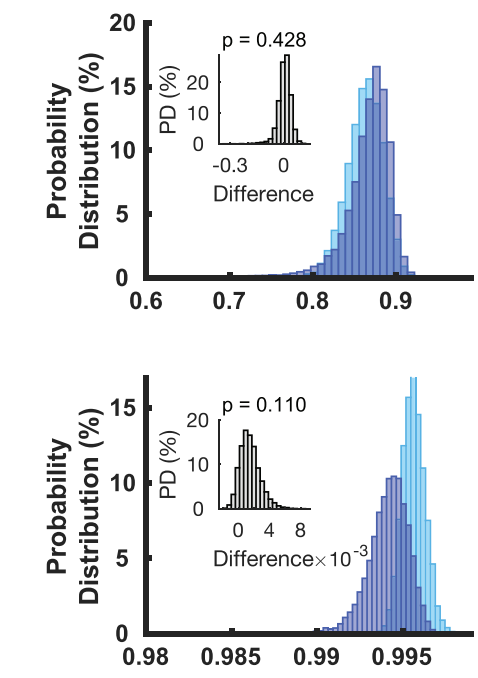

Slow Retention As

Fast Learning Rate Bf

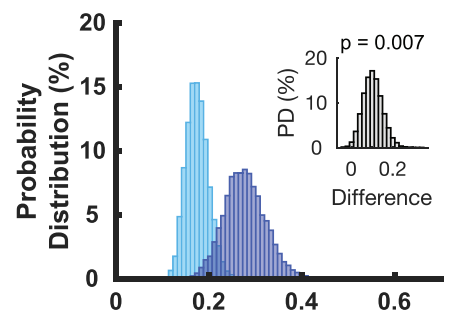
Learning Rate Bs

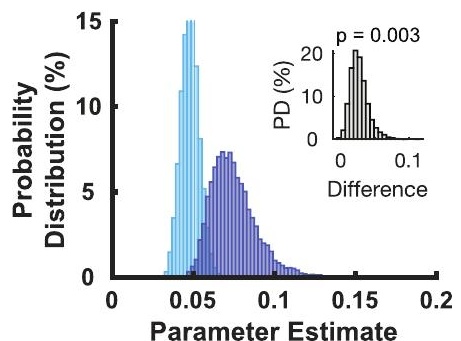

Control Session 1 vs Structural Learning Session 2
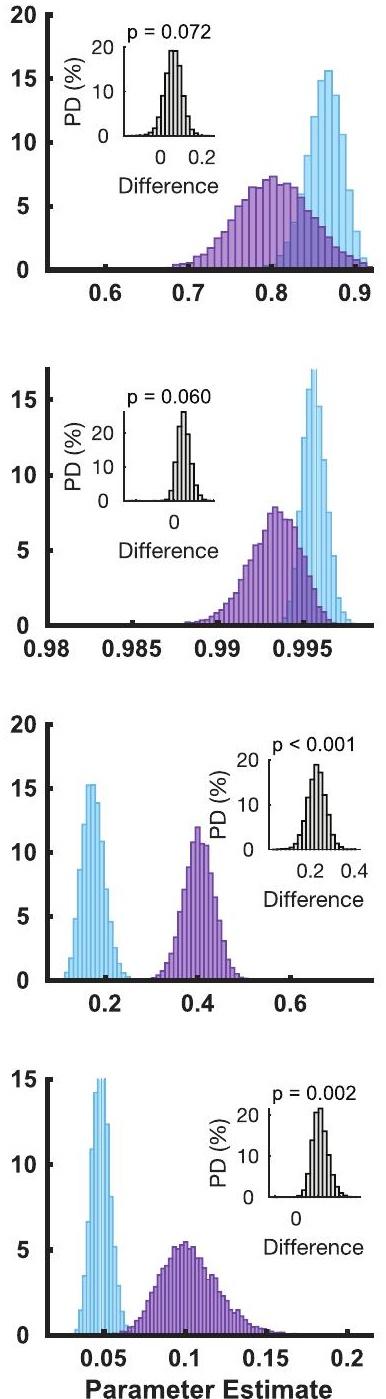

Control Session 1 vs

Gradual Learning Session 2
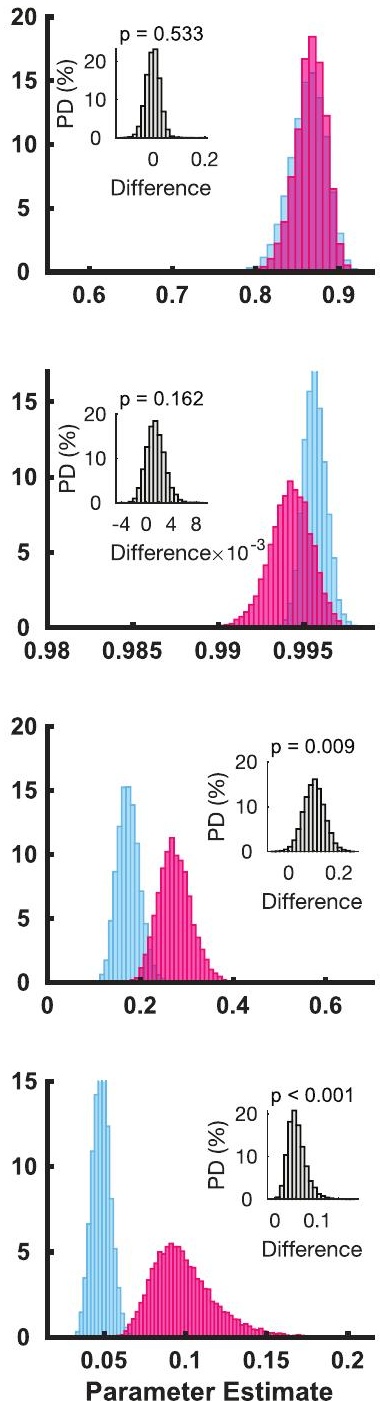

Structural Learning Session 2 vs

Gradual Learning Session 2
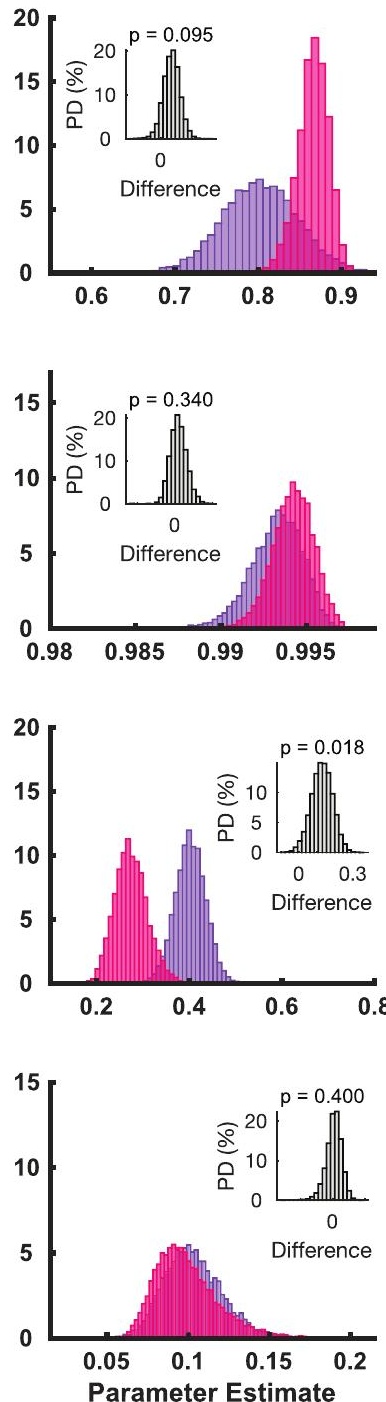
